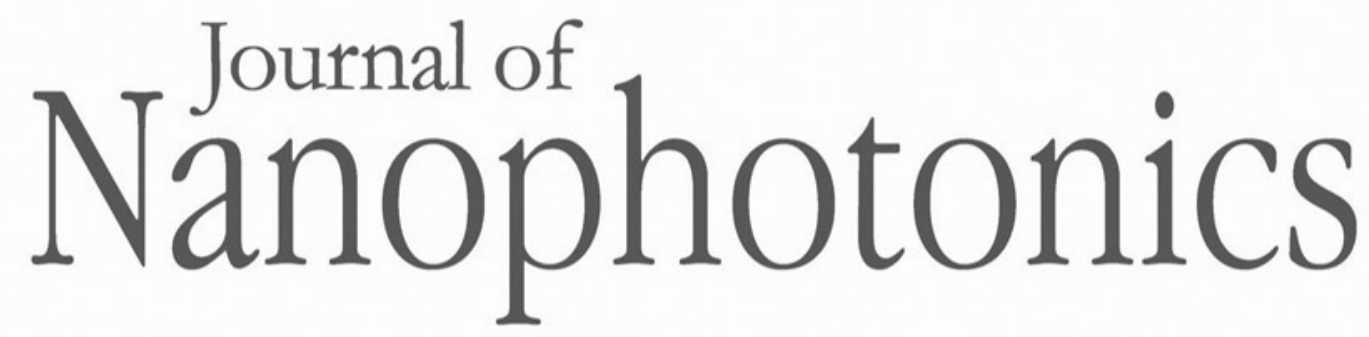

\title{
Twisted mass transport enabled by the angular momentum of light
}

\author{
Takashige Omatsu \\ Keigo Masuda \\ Katsuhiko Miyamoto \\ Kohei Toyoda \\ Natalia M. Litchinitser \\ Yoshihiko Arita \\ Kishan Dholakia
}




\title{
Twisted mass transport enabled by the angular momentum of light
}

\author{
Takashige Omatsu, ${ }^{\text {a,b, } * \text { Keigo Masuda, }}{ }^{\text {a }}$ Katsuhiko Miyamoto, ${ }^{\text {a,b }}$ \\ Kohei Toyoda, ${ }^{\text {a,b }}$ Natalia M. Litchinitser, ${ }^{c}$ Yoshihiko Arita, ${ }^{\text {b,d }}$ and \\ Kishan Dholakia ${ }^{\text {a,d,e }}$ \\ ${ }^{a}$ Chiba University, Graduate School of Engineering, Chiba, Japan \\ ${ }^{\mathrm{b}}$ Chiba University, Molecular Chirality Research Center, Chiba, Japan \\ ${ }^{c}$ Duke University, Department of Electrical and Computer Engineering, Durham, North \\ Carolina, United States \\ ${ }^{\mathrm{d}}$ SUPA, University of St. Andrews, School of Physics and Astronomy, Fife, United Kingdom \\ ${ }^{e}$ Yonsei University, College of Science, Department of Physics, Seoul, Republic of Korea
}

\begin{abstract}
Light may carry both orbital angular momentum (AM) and spin AM. The former is a consequence of its helical wavefront, and the latter is a result of its rotating transverse electric field. Intriguingly, the light-matter interaction with such fields shows that the orbital AM of light causes a physical "twist" in a range of materials, including metal, silicon, azopolymer, and even liquid-phase resin. This process may be aided by the light's spin AM, resulting in the formation of various helical structures. The exchange between the AM of light and matter offers not only unique helical structures at the nanoscale but also entirely novel fundamental phenomena with regard to the light-matter interaction. This will lead to the future development of advanced photonics devices, including metamaterials for highly sensitive detectors as well as reactions for chiral chemical composites. Here, we focus on interactions between the AM of light and azopolymers, which exhibit some of the most diverse structures and phenomena observed. These studies result in helical surface relief structures in azopolymers and will leverage next-generation applications with light fields carrying optical AM. (C) The Authors. Published by SPIE under a Creative Commons Attribution 4.0 Unported License. Distribution or reproduction of this work in whole or in part requires full attribution of the original publication, including its DOI. [DOI: 10.1117/1.JNP.14 .010901]
\end{abstract}

Keywords: singular optics; optical vortices; orbital angular momentum; spin angular momentum; laser materials processing; chiral structured materials; azopolymers.

Paper 19134SSV received Nov. 1, 2019; accepted for publication Feb. 25, 2020; published online Mar. 18, 2020.

\section{Introduction}

Light carries orbital angular momentum (AM) of $\ell \hbar$ per photon, associated with its helical wavefront and an azimuthal phase term is denoted by $\exp (i \ell \phi)$, where $\ell$ is an integer termed the topological charge or azimuthal index, and $\phi$ is the azimuthal angle. ${ }^{1-3}$ Typically, such fields also possess an on-axis phase singularity, termed an optical vortex. To date, the orbital AM of light has provided a variety of emerging applications at the nano-/microscale, such as optical trapping and manipulation, ${ }^{4-7}$ and optical microscopy with a spatial resolution below the diffraction limit. ${ }^{8-10}$ Further applications of optical vortices with orbital AM include quantum communications, ${ }^{11}$ fiber-based or free-space telecommunications, ${ }^{12,13}$ and environmental optics. Optical vortices themselves are a very broad and attractive field for a number of reasons, and they can propagate though turbulent air with lower beam degradation than a standard Gaussian beam. ${ }^{14,15}$

Recent light-matter interaction studies have shown a new fundamental physical phenomenon, namely that light fields carrying orbital AM can physically "twist" materials, such as metals, silicon, azopolymers, and liquid-phase photosensitive resins, forming helical nano-/ microstructures. This is often seen with the presence of additional spin AM, sh per photon

*Address all correspondence to Takashige Omatsu, E-mail: omatsu@ faculty.chiba-u.jp 
$(s= \pm 1)$, associated with the rotating electric field present in circularly polarized light. ${ }^{16-22}$ A total AM, $[J=(\ell+s)]$, of light refers to the sum of both the orbital and spin AM associated with both the beam's helical wavefront and polarization. ${ }^{23-25}$ This total AM has been experimentally observed in studies of optically trapped microparticles exhibiting orbital and spinning motion depending on the orbital and spin AM of the trapping beam. ${ }^{26-28}$

Separately, a pulsed orbital AM field has enabled the formation of nano-/microscale "twisted" needle-like structures (so-called twisted needles) on the surface of irradiated bulk tantalum (Ta), silicon (Si), copper (Cu) and silver (Ag), and gold (Au) thin films ${ }^{16-22,29,30}$ (Fig. 1). Such twisted needles can be formed via several thermodynamic steps, including the aggregation of melted material revolving at the dark center of the vortex beam through the effects of thermocapillary forces and Rayleigh-Plateau hydrodynamic instability.

Ultraviolet or visible continuous-wave (CW) light can induce polymerization of liquid-phase photosensitive resins (photopolymerization), creating 3D objects with a micrometer resolution. Photopolymerization can take a form of self-focusing and self-trapping creating a waveguiding fiber (the so-called "laser-induced self-written fiber"), which can extend to millimeter
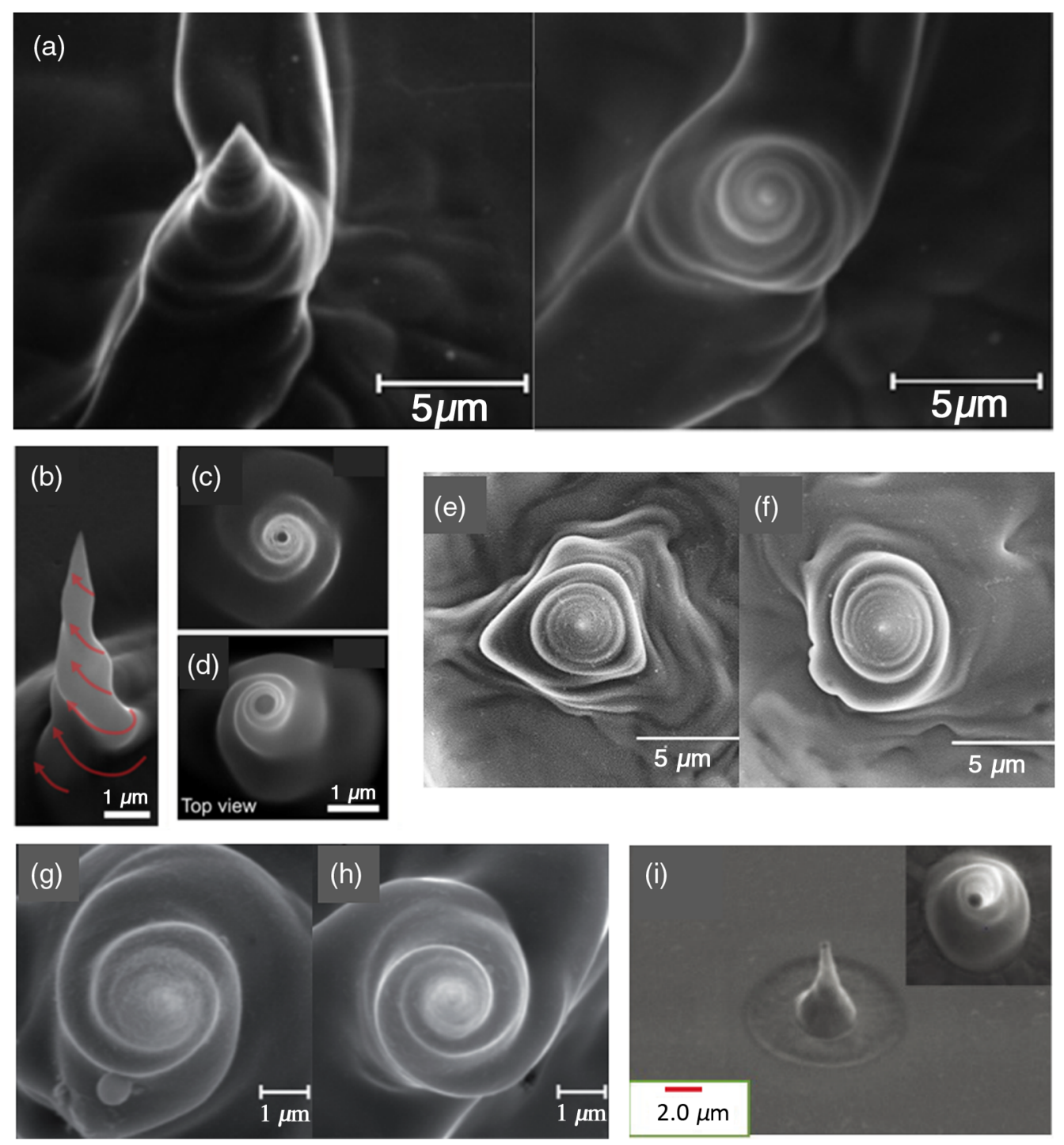

Fig. 1 Twisted needles fabricated on various metal substrates. (a) Ta substrate. ${ }^{16}$ (b)-(d) Ag film, ${ }^{20}$ (e), (f) Si substrate, ${ }^{21}$ (g), (h) Cu needles, ${ }^{29}$ and (i) Au thin film. ${ }^{30}$ Reproduced with permission. ${ }^{16,20,21,29,30}$ Copyright 2012, 2017, 2016, 2017, American Chemical Society, The Optical Society, Springer Nature, Institute of Physics, Japan Laser Processing Society. 
lengths. ${ }^{31,32}$ The photopolymerization of self-written fibers with an incident helical wavefront possessing orbital AM, instead of a conventional planar wavefront, has recently been investigated $^{33}$ (Fig. 2). The incident optical vortices with orbital AM of $\pm \hbar$ are focused into the resin, where fibers with helical features are formed along the beam propagation direction. Higher-order orbital AM beams also enable the formation of multiple microfibers with corresponding topological charges. Such helical fiber formation can be explained by spatial soliton effects (including self-focusing and self-trapping). ${ }^{34,35}$

Going beyond traditional research avenues using optical vortices and orbital AM, the formation of twisted materials with optical vortices carrying orbital AM should lead to new physical insights into the light-matter interactions at the nano-/microscale, including spin-orbit coupling of light ${ }^{36}$ and exotic optomechanical effects. ${ }^{37,38}$ The interplay between an orbital AM field and a judiciously chosen material can also offer new avenues for advanced photonic devices, ${ }^{39}$ such as metamaterials to sense and react with chiral chemical composites, ${ }^{40}$ and optical waveguides for optical vortex mode-based space-division multiplexing telecommunications. ${ }^{41}$

It should be further noted that near-field optical devices based on nano-/microstructured materials, such as metasurfaces, ${ }^{42}$ photonic/plasmonic crystals, ${ }^{43,44}$ and scanning near-field optical microscope probes, ${ }^{45}$ have advanced significantly in recent years, such that we may readily generate optical vortices with orbital AM at the nanoscale. ${ }^{46,47}$ Such near-field optical devices should further enable subwavelength-scale interaction between optical fields and matter beyond the diffraction limit.

Here, we focus on the interaction of light fields with orbital AM and azopolymers, which are seen to show some of the most striking, diverse structures and phenomena when considered alongside a wide variety of materials. Azobenzene exhibits polarization reversibility allowing
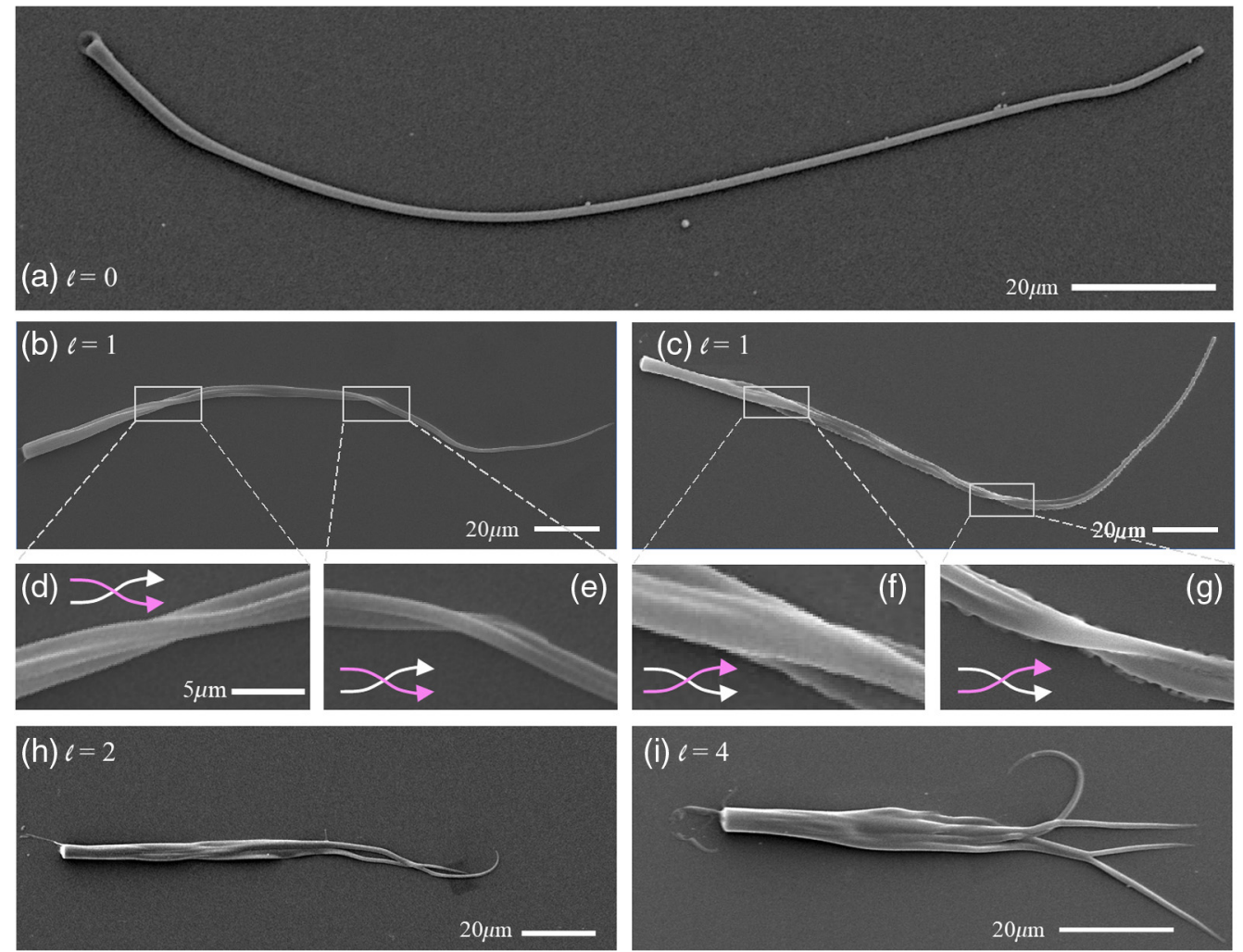

Fig. 2 SEM images of the photopolymerized fibers created by irradiation of a Gaussian beam with $\ell=0$ and LG beams with $|\ell| \geq 1$. (a) $\ell=0$ and (b) $\ell=1$. (c), (d) Expanded views of the selected areas in (d) showing the clockwise helix. (e) $\ell=-1$. (f), (g) Expanded views of the selected areas in (g) showing the counterclockwise helix. (h) $\ell=2$ exhibiting a fiber with two branches. (i) $\ell=4$ exhibiting four branches. The $20-\mu \mathrm{m}$ scale bar in panel (a) applies to (b), (e), (h), and (i), whereas the $5-\mu \mathrm{m}$ bar in panel (c) applies to (d), (f), and (g). Reproduced with permission. ${ }^{33}$ Copyright 2018, American Chemical Society. 
one to make photo-induced surface patterns. Typically, photoresists are used for mask creation and respond to the intensity of short-wavelength visible light. In contrast, azopolymers create masks via the process of modulation of the light intensity and polarization, which means extraneous light plays little to no role. Such azopolymers are responsive to a broad range of wavelengths in the visible region and are markedly better than photoresist when considering extended periods of exposure. They are a material of choice when considering the creation of more advanced, intricate patterning.

Irradiation by $\mathrm{CW}$ visible light for such materials enables the mass transport of azopolymers through trans-cis photoisomerization ${ }^{48,49}$ as well as photoinduced self-orientation, ${ }^{50}$ so as to form a surface relief. ${ }^{51}$ Such mass transport of azopolymers occurs mainly along the field intensity gradient of the illuminated light; however, it was recently discovered that the optical vortices with orbital AM induce the wavefront-sensitive helical mass transport of azopolymers, ${ }^{52-57}$ forming a helical surface relief. We will review novel structures and fundamental phenomena in azopolymers induced by the interaction between materials and light fields possessing spin AM or orbital AM or both forms of AM.

\section{Surface Relief Formation in Azopolymers}

Nonuniform illumination of azopolymers results in nonuniform trans-cis photoisomerization via light absorption, which results in spatially anisotropic photofluidity. ${ }^{51,58}$ Mass transport of azopolymers induced by the optical scattering force occurs subsequently from a bright region toward a dark region along the polarization direction of the illuminated light. This forms a surface relief on an azopolymer film that is indicative of the polarization state and spatial intensity profile of the incident light ${ }^{59-61}$ (Fig. 3).

In general, the trans-form azopolymer is thermodynamically stable in comparison with the cis-form azopolymer (energy barrier between trans- and cis-forms is $c a .50 \mathrm{~kJ} / \mathrm{mol}$ ). Thus, the trans-form azopolymers mostly survive at room temperature. Irradiation of light fields partially transforms light-irradiated azopolymers to the cis-form, and subsequently, the azopolymers revert to the trans-form by two processes: the spontaneous thermal-reaction and the cis-trans reverse photoisomerization via rotation or inversion ${ }^{62-65}$ (Fig. 4). Such trans-cis or cis-trans isomerization induces a spring-like motion of the azopolymer with an elongated trans-form and a contracted cis-form. ${ }^{48}$ Furthermore, azopolymers reorient along the direction perpendicular to the polarization direction of the incident light after light absorption. ${ }^{66}$ As a result, azopolymers move from a bright part of the beam to a dark part along the light polarization, that is, they

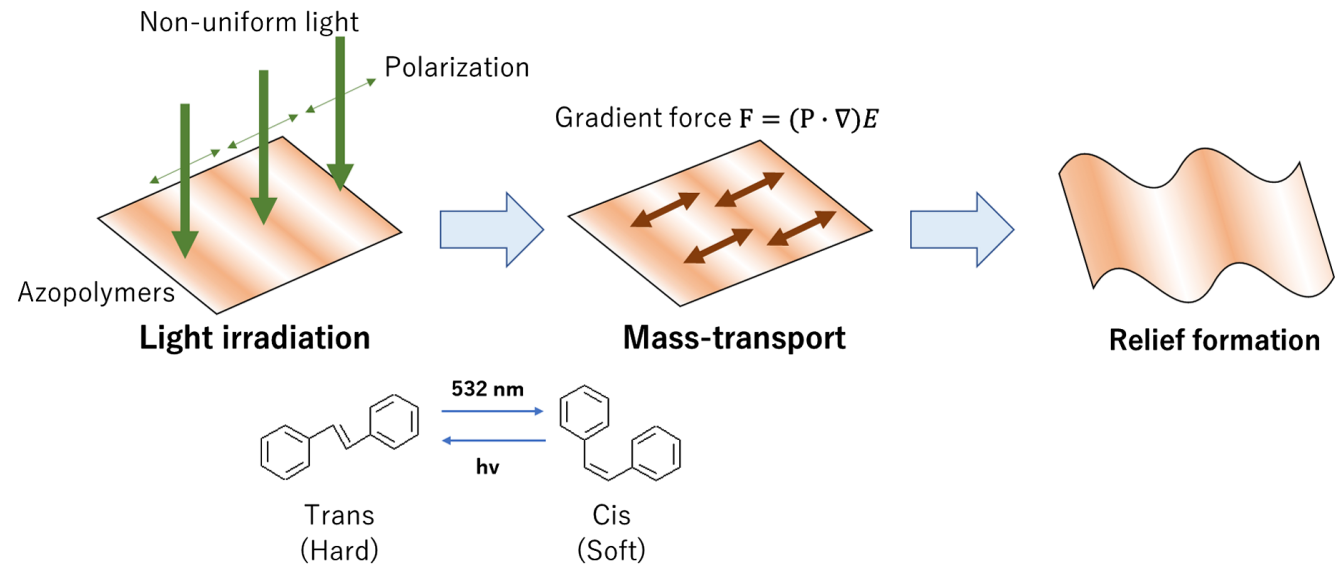

Fig. 3 Schematic surface relief formation in an azopolymer film. Nonuniform illumination on the film leads to nonuniform trans-cis photoisomerization via light absorption, resulting in spatially anisotropic photofluidity. Mass transport of the azopolymers occurs subsequently by the optical scattering force from a bright region towards a dark region along the polarization direction of the illuminated light, thereby forming a surface relief on an azopolymer film. This relief manifests the polarization state and spatial intensity profile of the incident light. 


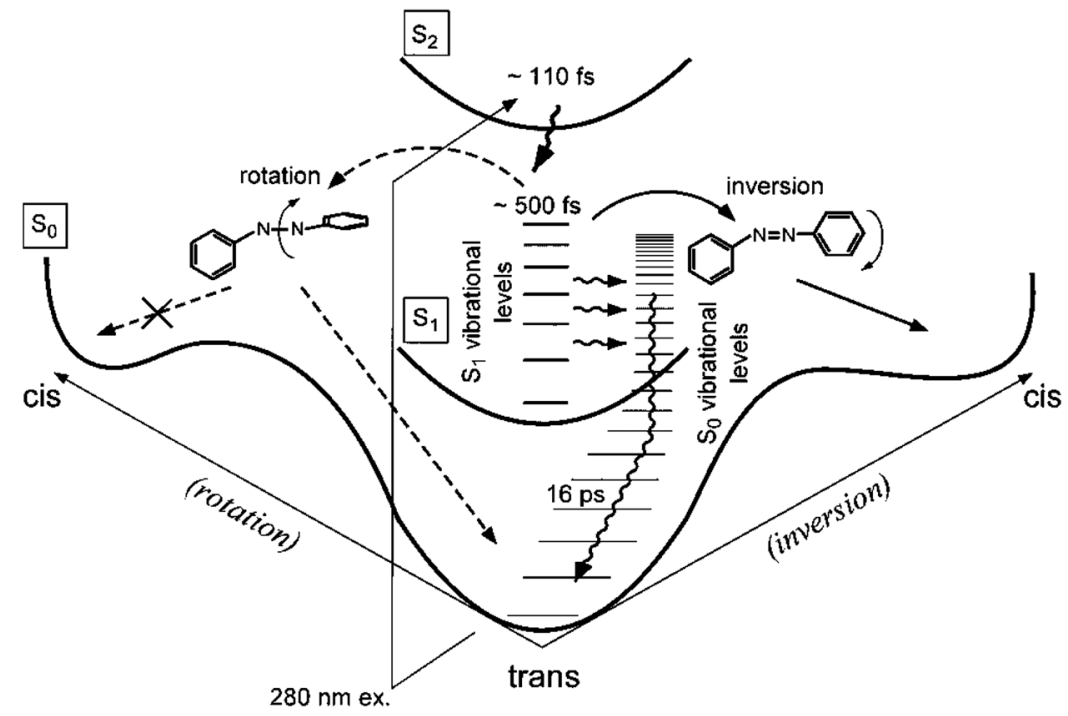

Fig. 4 Schematic diagram of the relaxation and isomerization pathway of trans-azobenzene after the $\mathrm{S} 2(\pi \pi *)$. Reproduced with permission. ${ }^{65}$ Copyright 2001, American Chemical Society.

exhibit light-induced mass transport. Furthermore, interference patterns induced by the incident light fields can form surface relief gratings on an azopolymer film, which, in turn, enables the development of optical devices, such as holograms, waveguide couplers, and photonic waveguide circuits (Fig. 5).

A number of theoretical models have been proposed for the light-induced mass transport of azopolymers. Kumar et al. reported the mechanism due to interaction between oriented azopolymers where considered as dipoles and the optical electric field gradient. ${ }^{49,67}$ Barrett et al. proposed a light-induced pressure model. ${ }^{68}$ Lefin et al. and Bellini et al. introduced a diffusion model based on random walk motion. ${ }^{69,70}$ A mean-field theory model ${ }^{71}$ and a fluid mechanics model ${ }^{72,73}$ have also been proposed to date. Sekkat further proposed a model based on the photoisomerization force and the optical gradient force. ${ }^{48}$

A tightly focused single laser beam can also induce the outward (or inward) mass transport of azopolymers along the polarization direction of the incident light, thus making a dip (or bump) on the focus point ${ }^{74,75}$ (Fig. 6). Sekkat's model considers the polymers as a viscous liquid with fluidic motion, where the forces of photoisomerization, optical gradient and elastic restoration, due to the elasticity of the polymers themselves, all drive the mass transport of azopolymers. ${ }^{48}$ The inward (or outward) mass transport toward (or from) an optical field by irradiation with a single laser beam is determined by the sum vector of the optical gradient force, the elastic force,
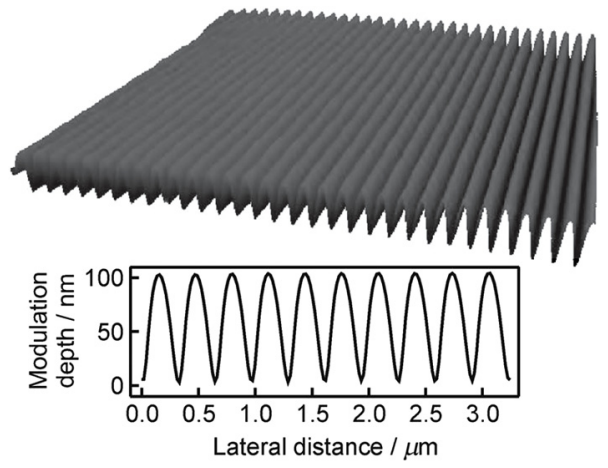

Fig. 5 Atomic force microscopy image (top) and surface profile of relief patterns in an azopolymer film (bottom) with a period of $320 \mathrm{~nm}$ and surface-modulation depth of $100 \mathrm{~nm}$. Reproduced with permission. ${ }^{61}$ Copyright 2011 , Wiley. 

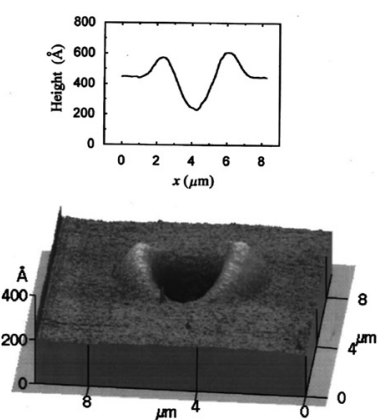

(a)
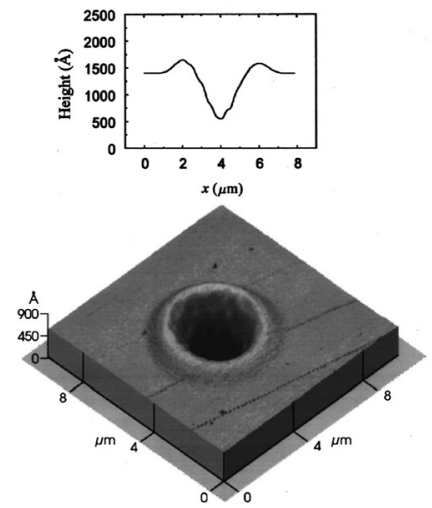

(b)

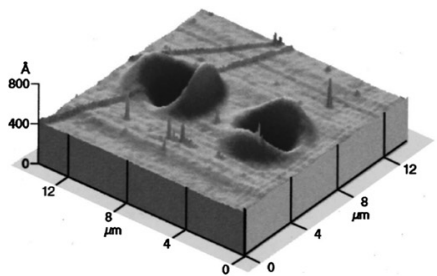

(c)

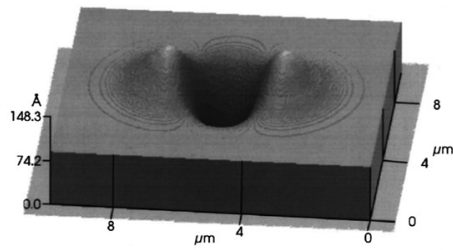

(d)

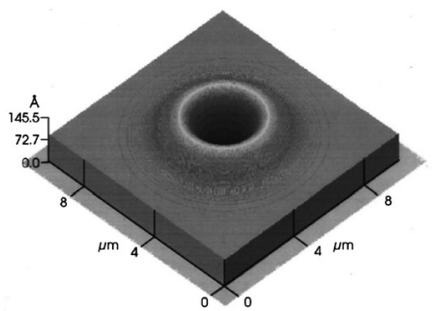

(e)

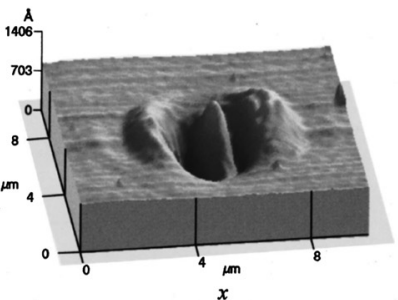

(f)

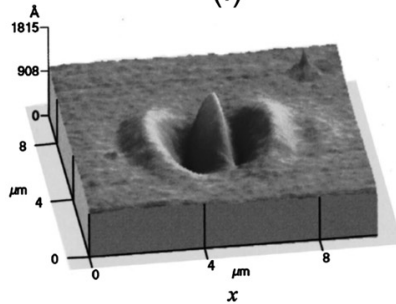

(g)

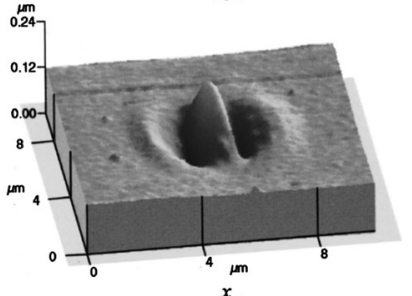

(h)

Fig. 6 Surface reliefs induced by (a) linearly polarized (along the $x$ axis) Gaussian beam, (b) circularly polarized Gaussian beam, and (c) two Gaussian beams with orthogonal linear polarization. The inset in (a) and (b) shows the surface profile, respectively. Theoretically calculated surface deformation for Gaussian beam with (d) linear and (e) circular polarization. Irradiated laser intensity was then $\sim 5 \mathrm{~W} / \mathrm{cm}^{2}$. Surface reliefs induced by $(\mathrm{f})-(\mathrm{h})$ linearly polarized (along the $x$ axis) Gaussian beam with high intensity of (f) $\sim 5.3 \mathrm{~kW} / \mathrm{cm}^{2},(\mathrm{~g}) \sim 5.5 \mathrm{~kW} / \mathrm{cm}^{2}$, and $(\mathrm{h})$ $\sim 5.9 \mathrm{~W} / \mathrm{cm}^{2}$. Reproduced with permission. ${ }^{75}$ Copyright 1999, American Institute of Physics.

and the photoisomerization force. Bian et al. suggest that the inward mass transport of azopolymers typically occurs by irradiation of laser beam with high intensity, and it may be due to the photothermal effects and photochemical degradation/bleaching. ${ }^{75}$

However, it is fair to say a comprehensive picture of the phenomenon of light-induced mass transport of azopolymers is still under debate and this includes the role of many complicated photochemical processes, such as photoisomerization, photodegradation, and photobleaching, as mentioned above.

\section{Orbital AM Induced Surface Relief}

\subsection{Theoretical Model}

There have only been a few theoretical studies to date as to how an azopolymer film interacts with an optical field with orbital AM. Ambrosio et al. proposed a macroscopic simulation model for wavefront-sensitive mass transport induced by optical vortex. In this model, light-induced mass transport is determined by a mass-current-density vector, i.e., mass-flow rate vector, which is determined by the optical field (and its gradient), and other contributions such as viscouselastic interactions. ${ }^{56}$ The authors also assume that interference between the transverse and the longitudinal optical electric fields leads to the material possessing rotational symmetry breaking owing to the presence of a surface discontinuity of the medium. Their model can explain the double-armed surface relief formation in the azopolymers with the use of a tightly focused optical system. They further proposed a detailed microscopic mechanism, in which trans- (or cis) 
azopolymer is assumed to act as a dipole, where the anisotropic molecular diffusion is enhanced in the proximity of the free-polymer surface. ${ }^{57}$ However, their models do not allow us to directly understand the mechanism of wavefront-sensitive surface relief formation in an azopolymer upon irradiation with optical vortices. As a result, it is also difficult to understand how the spin AM and orbital AM interact to contribute to the wavefront-sensitive surface relief formation.

Barada et al. described a simple analytical model for the optical radiation force induced by a time-averaged orbital AM light field in an isotropic and homogeneous medium within the paraxial approximation. ${ }^{53}$ Their simple model provides a direct understanding of the surface relief formation by irradiation of optical vortices carrying an arbitrary topological charge and polarization state. The radiation force $\mathbf{F}$ can be written in the form of

$$
\begin{aligned}
& \mathbf{F}(r, \phi, z) \propto \chi_{r}\left[\frac{\sqrt{1-s^{2}}}{2} \frac{\partial A_{\ell}^{2}}{\partial r}\left[-\cos (\phi) \cdot e_{x}+\sin (\phi) \cdot e_{y}\right]+\left(\frac{1}{2} \frac{\partial A_{\ell}^{2}}{\partial r}-\ell s \frac{A_{\ell}^{2}}{r}\right) \cdot e_{r}\right] \\
& \quad+\chi_{i}\left[\ell \frac{\sqrt{1-s^{2}}}{2} \frac{A_{\ell}^{2}}{r}\left[\sin (\phi) \cdot e_{x}+\cos (\phi) \cdot e_{y}\right]+\left(\ell \frac{A_{\ell}^{2}}{r}-\frac{s}{2} \frac{\partial A_{\ell}^{2}}{\partial r}\right) \cdot e_{\phi}\right]+2 k A_{\ell}^{2} \cdot e_{z},
\end{aligned}
$$

where $e_{r}, e_{\phi}$, and $e_{z}$ are the unit vectors along the $r, \phi$, and $z$-axes, respectively; $e_{x}$ and $e_{y}$ are also unit vectors for the polarization state in a Cartesian coordinate system [see Fig. 7(a)]; $A_{l}(r)$ is the radial amplitude of the optical vortex field; $k$ is the wavenumber; and $\chi$ $\left(=\chi_{r}+i \chi_{i}=2+2 i\right)$ is the macroscopic complex electric susceptibility of the material.

The radial amplitude $A_{l}(r)$ can be explicitly written as

$$
A_{\ell}(r)=\frac{A_{0}}{\sqrt{\ell !}}\left(\sqrt{2} \frac{r}{\omega_{0}}\right)^{|\ell|} \exp \left(-\frac{r^{2}}{\omega_{0}^{2}}\right) A_{\ell}(r)
$$

where $\omega_{0}$ is the beam waist of the optical vortex.

The optical field carries the same handedness of orbital AM and spin AM, i.e., both positive orbital AM and spin AM or vice versa, which creates a restoring force in the radial direction to accumulate materials in the dark core of the vortex beam. In the azimuthal direction, there is a force driving the orbital motion of the materials toward the clockwise (counter-clockwise) direction around the dark vortex core. On the other hand, when the optical field yields the opposite handedness of orbital AM and spin AM, i.e., positive orbital AM and negative spin AM or vice versa, the optical vortex produces a repulsive radial force, which prevents the accumulation of the materials in the dark core of the vortex beam. The azimuthal force, in this case, reverses inside and outside the dark core, which also prevents the smooth orbital motion of the azopolymers $^{53}$ (Fig. 7).

These results indicate the breaking of the degeneracy among optical fields with the same total $\mathrm{AM}$, expressed as the fourth term $\left(-\chi_{r} \cdot \ell \cdot s \cdot \frac{A_{t}^{2}}{r} \cdot e_{r}\right)$ in Eq. (1): the conclusion is that only optical fields with the same handedness of orbital AM and spin AM lead to the formation of helical microstructures.

\subsection{Helical Surface Relief}

Ambrosio et al. discovered that tightly focused laser beams carrying higher-order orbital AM modes can induce mass transport of azopolymers in the azimuthal direction forming a shallow "spiral-relief" with a depth of 10 to $20 \mathrm{~nm}^{56}$ (Fig. 8). They used a 700-nm-thick azopolymer film spin-coated onto a microscope coverslip. Due to azo groups, the film exhibited a broad absorption band around the visible wavelengths, with 5\% absorption at the wavelength of $532 \mathrm{~nm}$. A linearly polarized 532-nm beam carrying orbital AM with a topological charge $\ell=10$ was tightly focused to an annular spot of a few micrometers in size on the film surface by a high numerical aperture $(\mathrm{NA})$ objective lens $(\mathrm{NA}=1.3)$. The exposure time was $90 \mathrm{~s}$ with the beam power of $\sim 5 \mu \mathrm{W}$. A two-armed spiral surface relief was formed on the film due to the wavefrontsensitive mass transport of azopolymers. As predicted, the spiral relief direction was reversed without any change of the relief pattern by inverting the sign of the topological charge, $\ell$. 


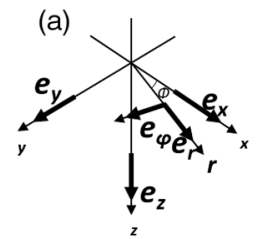

(b)

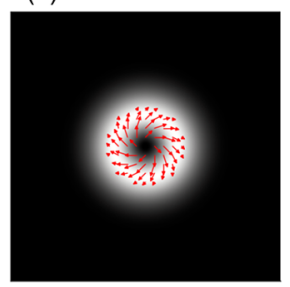

(c)

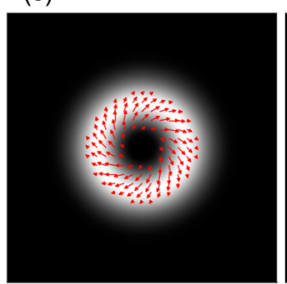

(e)

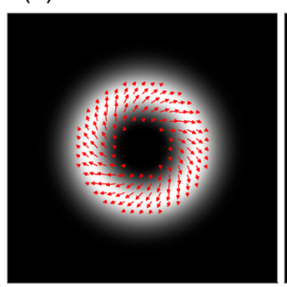

(h)

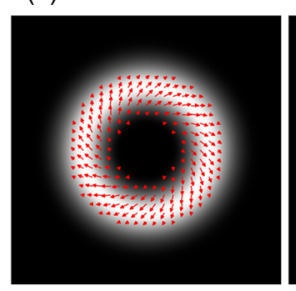

(d)

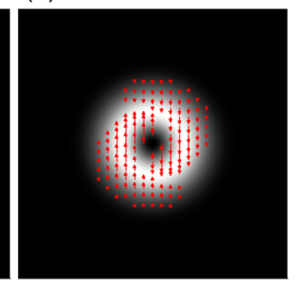

(f)

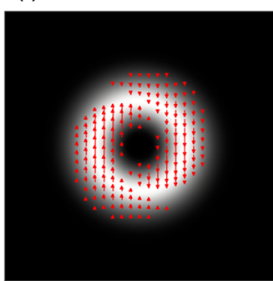

(i)

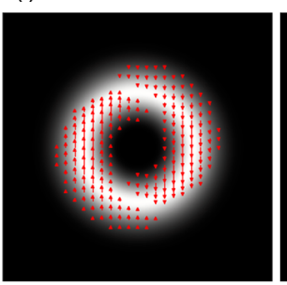

(g)

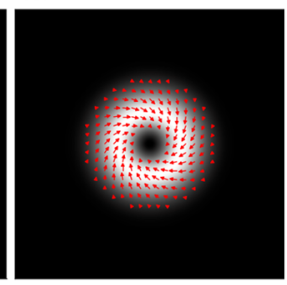

(j)

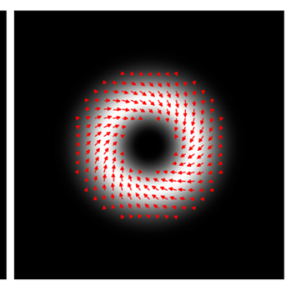

Fig. 7 Theoretical spatial distributions of the radial and azimuthal optical radiation forces produced by orbital AM beams with total angular momenta. (a) Schematic diagram of the coordinate systems for polarization unit vectors $\left(e_{x}, e_{y}\right)$, and radiation force unit vectors $\left(e_{r}, e_{\phi}\right.$, and $\left.e_{z}\right)$. $^{53}$ (b) $J=0(\ell=1, s=-1)$, (c) $J=1(\ell=2, s=-1)$, (d) $J=1(\ell=1, s=0)$, (e) $J=2(\ell=3$, $s=-1)$, (e) $J=2(\ell=2, s=0)$, (f) $J=2(\ell=1, s=1)$, (g) $J=3(\ell=4, s=-1)$, (h) $J=3$ $(\ell=3, s=0)$, and (i) $J=3(\ell=2, s=1)$. The optical field of the orbital AM beam, shown in white, underlies the optical radiation force.

Such wavefront-sensitive mass transport of azopolymers along the azimuthal direction manifests the existence of the interference between the longitudinal and transverse orbital AM fields, which is supported by their proposed models (Fig. 9).

Watabe et al. also demonstrated the formation of microscale chiral surface relief on the azopolymer film (termed "conch-shaped relief" in their work) by employing a lower-order orbital AM field with $\ell=1$ to 2 with spin $\mathrm{AM}^{52}$ (Fig. 10). A circularly polarized $532 \mathrm{~nm}$ first-order orbital AM beam was focused on the film by a relatively low NA objective lens $(\mathrm{NA} \approx 0.6)$, where the interference between the longitudinal and transverse orbital AM fields was negligible and the paraxial approximation remained valid. The azopolymer used was poly-orange-Tom1, and it exhibited a strong absorption band in the wavelength region of $300-550 \mathrm{~nm}$. 


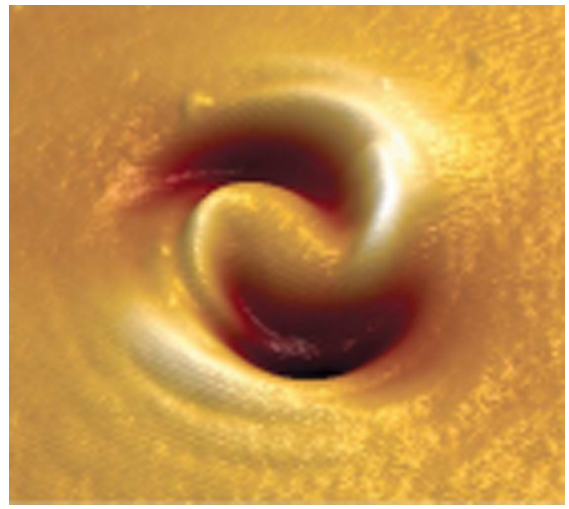

Fig. 8 Shallow "spiral relief." The 3D image of the surface relief created on an azopolymer thin film by illumination of an orbital AM beam with $\ell=10$. Reproduced with permission. ${ }^{56}$ Copyright 2012, Springer Nature.
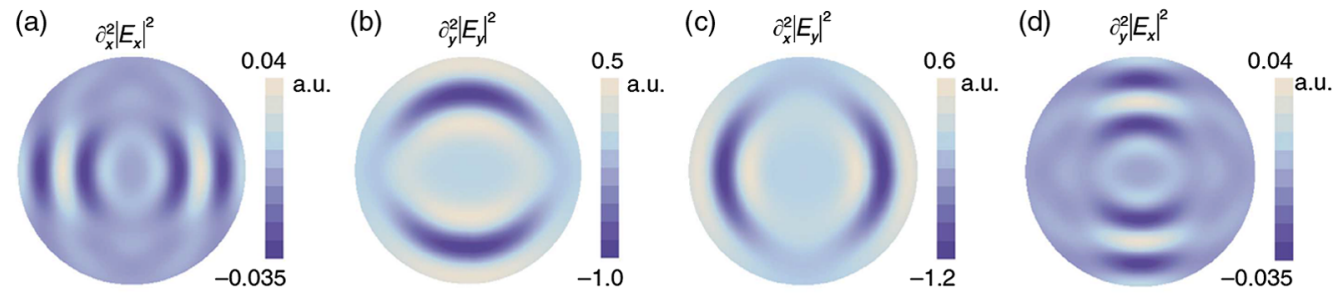

(e) $\partial_{x} \partial_{y}\left(E_{y}^{*} E_{x}+E_{x}^{*} E_{y}\right)$

(f) $\quad \hat{o}_{x}^{2}\left|E_{z}\right|^{2}$

(g) $\quad \partial_{y}^{2}\left|E_{z}\right|^{2}$
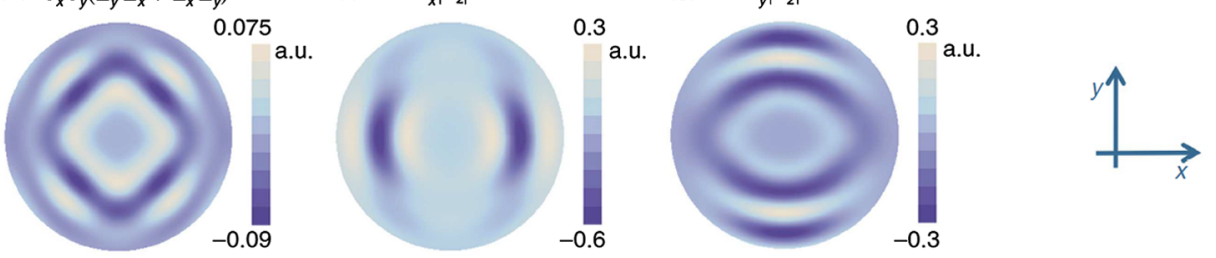

(h) $\partial_{x} \operatorname{Re}\left(E_{z}^{*} E_{x}\right)$

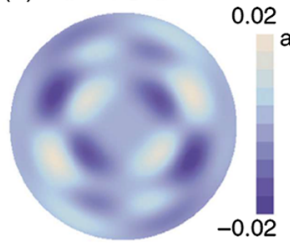

(i) $\partial_{y} \operatorname{Re}\left(E_{z}^{*} E_{y}\right)$
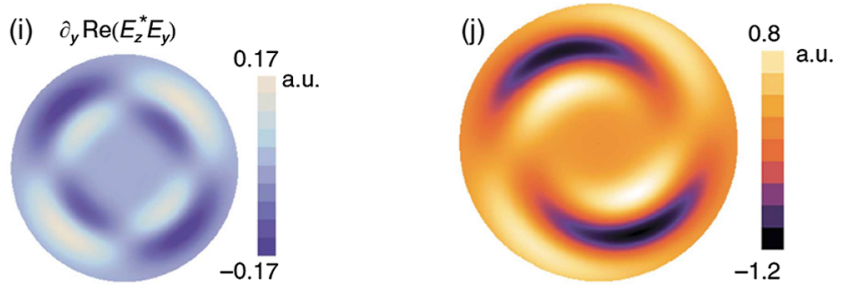

(k) $\partial_{x} \operatorname{Re}\left(E_{z}^{*} E_{x}\right)$

(l) $\partial_{y} \operatorname{Re}\left(E_{z}^{*} E_{y}\right)$


Fig. 9 Surface reliefs calculated by theoretical models. (a)-(i), (k), (l) Surface reliefs corresponding to the various terms appearing in our model. (j), (m) Surface reliefs obtained by combining all terms at the topological charges of \pm 5 . Reproduced with permission. ${ }^{56}$ Copyright 2012, Springer Nature. 
(a)

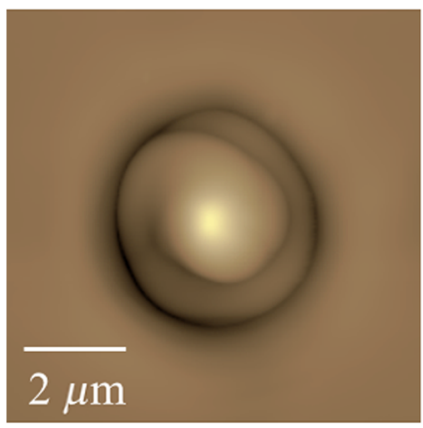

(c)

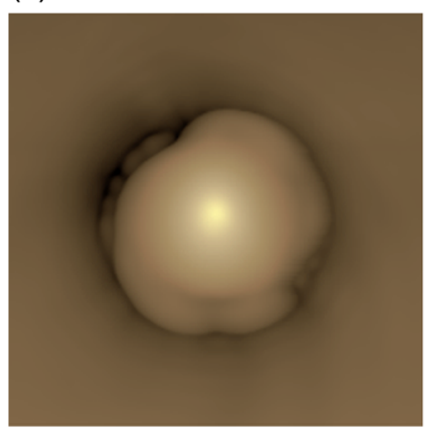

(b)

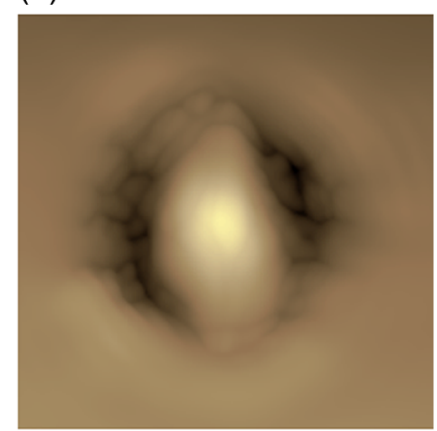

(d)

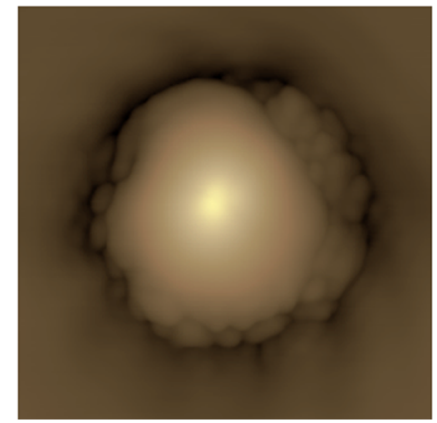

Fig. 10 Helical surface relief with a micron-scale depth. (a) and (b) Surface reliefs formed by irradiation of orbital AM beam with $J=2(\ell=1, s=1)$ and $J=2(\ell=2, s=0)$ respectively. (c), (d) Surface reliefs formed by orbital AM beam with $J=0(\ell=1, s=-1)$ and $J=0(\ell=-1$, $s=1)$, respectively. Reproduced with permission. ${ }^{52}$ Copyright 2014 , Springer Nature.

It should be noted that only circularly polarized beams with optical vortex carrying both positive (negative) orbital AM and positive (negative) spin AM can create a wavefront-sensitive helical surface relief. On the other hand, beams with opposite signs of orbital AM and spin AM (e.g., positive orbital AM and negative spin AM or vice versa) cannot form a helical structure, despite the fact it possesses nonzero total AM. Thus, the degeneracy among orbital AM fields with the same total AM is broken in the formation of surface relief. This degeneracy breaking originates from an inhomogeneous spatial density of the AM of the optical vortex field, which is predicted by the analytical formula proposed by Barada et al. for the optical radiation force induced by the time-averaged orbital AM field.

\subsection{Spin-Orbital AM Coupling}

It is well known that when a circularly polarized beam is tightly focused with an NA $>0.8$, a longitudinal electric field carrying a helical azimuthal phase can be produced in an isotropic and homogeneous medium (spin-orbital AM coupling). ${ }^{76,77}$ This allows the formation of helical surface relief on an azopolymer film with a circularly polarized Gaussian beam without orbital AM when tightly focused. However, this spin-orbital AM coupling can occur only in an isotropic and homogeneous azopolymer film. As soon as this helical surface relief is formed, the spatial symmetry of the azopolymer film will be broken (i.e., it becomes anisotropic). As a result, the spin-orbital AM coupling disappears. Thus, further exposure can transform the helical surface relief into a nonhelical structure ${ }^{54}$ (Fig. 11).

In recent years, Nakata et al. showed that rapid fabrication of multiple helical surface reliefs on an azopolymer film using an interference pattern formed by circularly polarized multiplexed beams $\left(488 \mathrm{~nm}\right.$, single-mode, $\mathrm{CW}$ ) with spin AM. ${ }^{78}$ An array of surface reliefs with a diameter of $5.6 \mu \mathrm{m}$ and a height of $1.1 \mu \mathrm{m}$ was formed in a regular triangle lattice (lattice constant: $11.8 \mu \mathrm{m}$ ) on an azopolymer film (Fig. 12). 


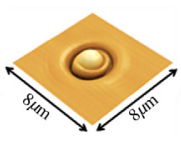

(a)

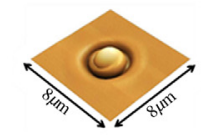

(b)
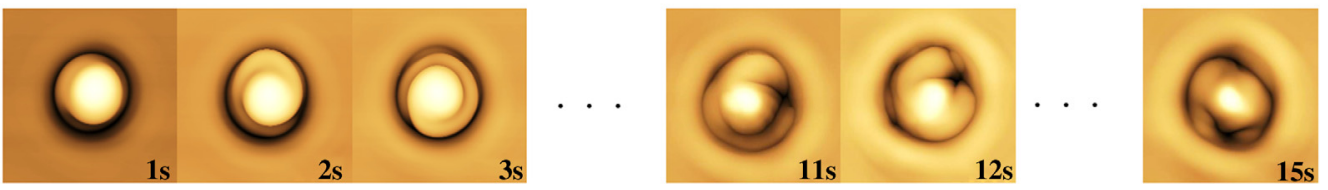

(c)
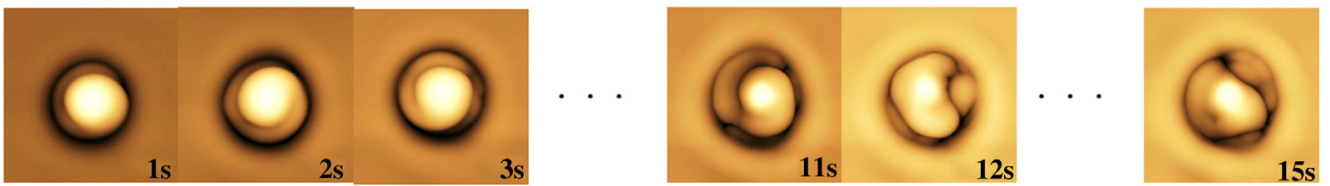

(d)
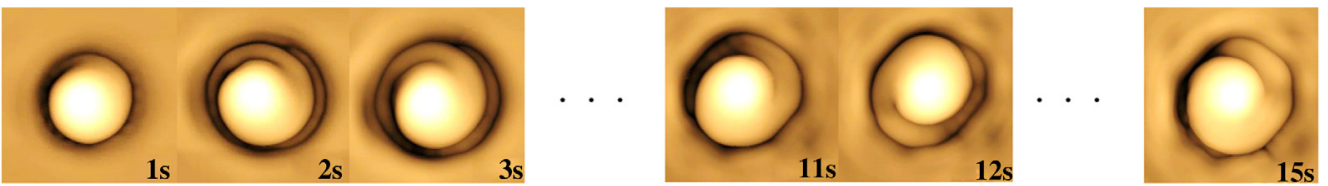

(e)

Fig. 11 Helical surface reliefs formed by (a) right- and (b) left-handed circularly polarized Gaussian beam illumination. Temporal evolution of the (c) right- and (d) left-handed surface relief formation. (e) Right-handed helical surface relief formation formed by illumination of circularly polarized orbital AM beam with $J=2$. Reproduced with permission. ${ }^{54}$ Copyright 2017, The Optical Society.

(a)

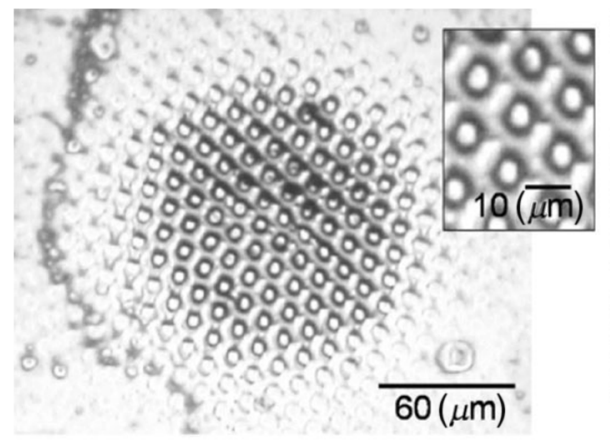

(b)

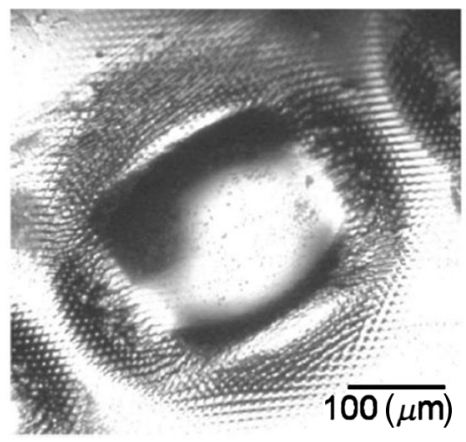

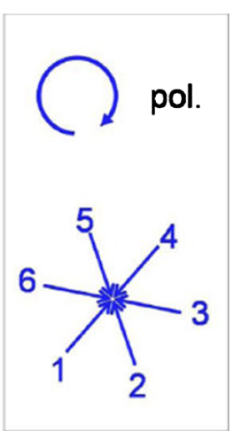

Fig. 12 Multiple surface reliefs fabricated by an interference pattern of six circularly polarized laser beams: (a) $P=35.8 \mathrm{~mW}$ and $\Delta t=6.0 \mathrm{~s}$, (b) $P=71.6 \mathrm{~mW}$ and $\Delta t=10.0 \mathrm{~s}$. Reproduced with permission. ${ }^{78}$ Copyright 2018, Springer Nature.

\subsection{Nanoscale Mass Transport}

Does such mass transport in azopolymers occur at a nanoscale, that is, beyond the diffraction limit? Two approaches exist: a multiphoton absorption process induced by ultrafast laser pulse or the use of the optical near-field. Two-photon-absorption-induced photoisomerization of azopolymers has been widely investigated ${ }^{79}$ in various fields, such as 3D optical data storage based on strong birefringence due to the light-induced molecular reorientation of azopolymers. ${ }^{80}$ However, most studies concerning surface relief formation in azopolymers are still based on single-photon-absorption, and there are only a limited number of the studies with the twophoton-absorption process. 

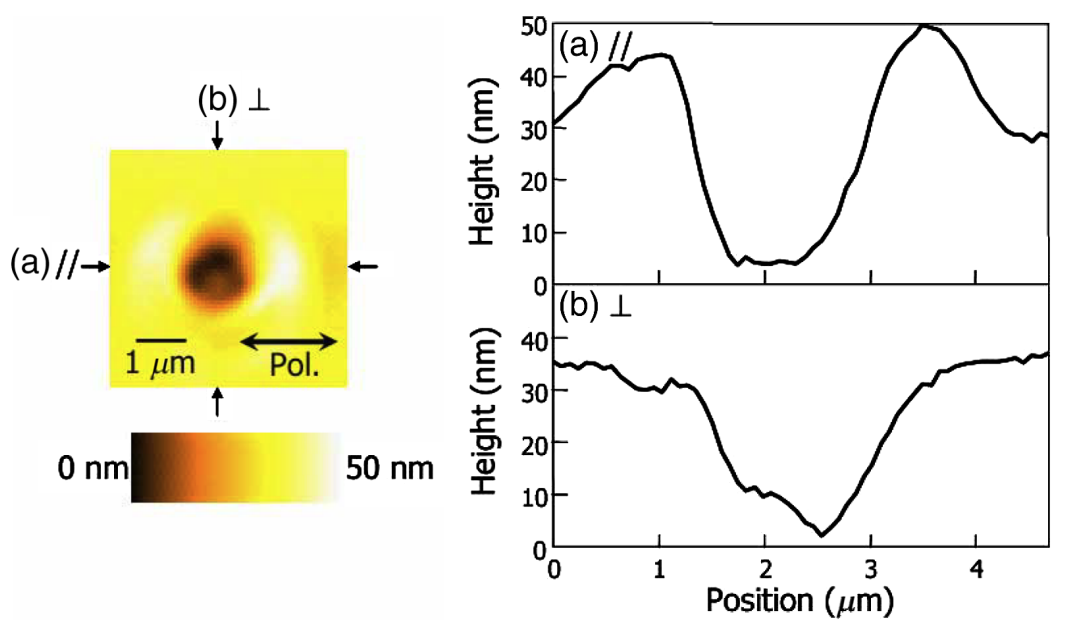

Fig. 13 Two-photon absorption-induced surface relief formed on an azopolymer film by irradation of 920-nm femtosecond laser. (a), (b) Line plots of the relief along the parallel and perpendicular direction to light polarization. Reproduced with permission. ${ }^{81}$ Copyright 2008 , The Optical Society.

Ishitobi et al. reported on surface relief structures induced by the two-photon-absorption process in an azo dye film. ${ }^{81}$ A 150 -nm-thick azopolymer film spin-coated on a glass substrate exhibited a strong absorption band at around the wavelength of $460 \mathrm{~nm}$. A linearly polarized femtosecond laser (wavelength: $920 \mathrm{~nm}$, pulse width: $130 \mathrm{fs}$, pulse repetition rate: $80 \mathrm{MHz}$ ) was focused to a $2-\mu \mathrm{m}$ spot on the film surface with an objective lens $(\mathrm{NA}=0.55)$ through the bottom substrate. The light intensity and exposure time were measured to be $61 \mathrm{~kW} / \mathrm{cm}^{2}$ and $60 \mathrm{~s}$, respectively. Nanoscale outward mass transport of the azopolymers occurred at the focused spot due to two-photon isomerization, which yielded a slightly elliptical dip (depth of ca. $40 \mathrm{~nm}$ ) with two side lobes along the polarization direction (Fig. 13). They also reported a photobleaching effect on the surface relief induced by irradiation of a 780-nm wavelength femtosecond laser.

Ambrosio et al. demonstrated the formation of surface relief in azopolymer films using the optical near-field ${ }^{82}$ with a spin-coated 550/50PMA/PMA4 copolymer film [thickness: $200 \mathrm{~nm}$, glass transition temperature $\left.\left(T_{g}\right): 294 \mathrm{~K}\right]$. The $417-\mathrm{nm} \mathrm{CW}$ laser was coupled into a metalized conical fiber tip (injected laser power: $0.1 \mathrm{~mW}$, nominal aperture: $50 \mathrm{~nm}$ ) placed at a distance of $10 \mathrm{~nm}$ from the sample. The height of the relief was measured to be $80 \mathrm{~nm}$ with the exposure time of $20 \mathrm{~s}$. This was well predicted by a formula derived from the Navier-Stokes equations for classical laminar flow (Fig. 14).

Karageorgiev et al. realized the isotropic viscous-elastic fluidization of an azopolymer film below $T_{g}$ using optical near-field illumination. The optical gradient force and van der Waals force were both exerted by a metallic tip, which played a key role in photoisomerization of the azopolymers. ${ }^{83}$ Such deformation of azopolymers should mimic the spatial profile of an optical electric field in the near-field. For example, a plasmon enhanced near-field in the vicinity of a single gold nanoparticle (diameter: 50 or $80 \mathrm{~nm}$ ) was visualized by the nanoscale deformation of an azopolymer thin film (thickness: 30 or $50 \mathrm{~nm}$; Fig. 15). ${ }^{84}$

\subsection{Nanoscale Helical Surface Relief}

The evanescent near-field generated at the interface between different materials typically exhibits spin AM-induced orbital AM, i.e., the generation of near-field orbital AM light. For instance, a helical surface relief structured at the interface between air and a thin gold film should play a role in the generation of near-field orbital AM light on a subwavelength scale by illumination of a circularly polarized beam with planar wavefronts.

Such near-field orbital AM light will lead to further nanoscale helical mass transport of azopolymers as seen by Masuda et al., who demonstrated the formation of a nanoscale helical surface relief by the near-field orbital AM light. ${ }^{55}$ The whole process had three steps: fabrication of a chiral surface relief by illumination of an optical vortex; creation of the Au coated chiral relief 
(a)

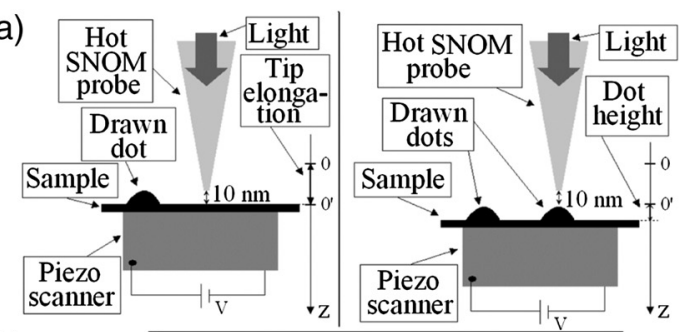

(b)

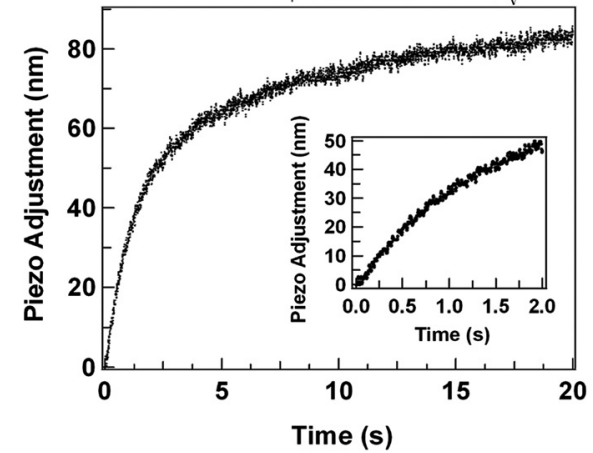

(c)

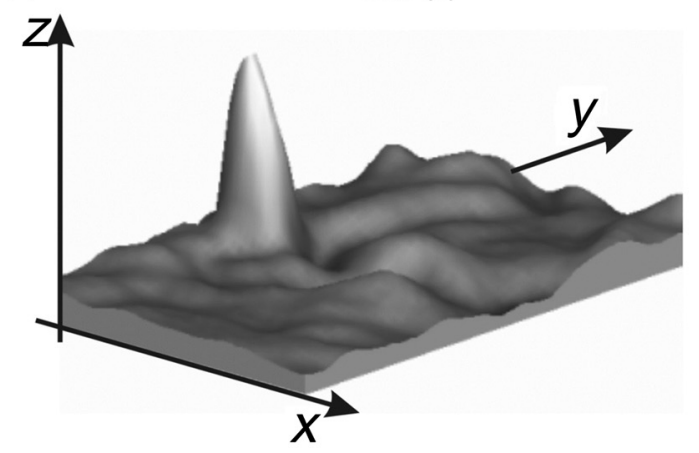

Fig. 14 Light-injected near-field optical microscope probe scanned on the azopolymer film. (a) Schematic of the data acquisition procedure. (b), (c) A dot is formed on the film surface. The dot height is about $65 \mathrm{~nm}$ and the diameter is of about $200 \mathrm{~nm}$. Reproduced with permission. ${ }^{82}$ Copyright 2008, Wiley.
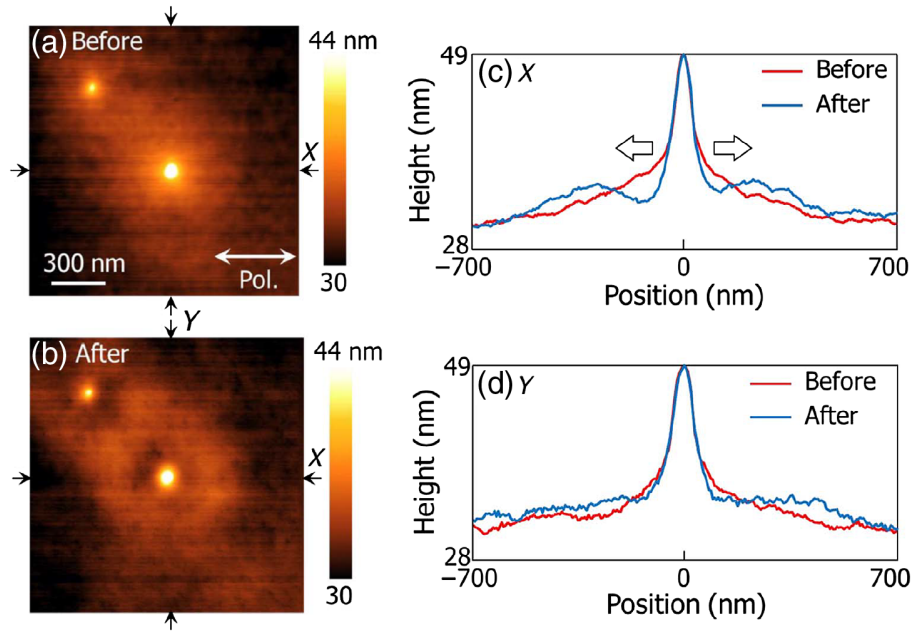

Fig. 15 Surface reliefs (a) before and (b) after induction by a plasmonically enhanced near-field in the vicinity of a 50-nm Au nanoparticle covered by an azopolymer on a glass substrate. White arrows indicate the light polarization. (c), (d) Line plots of the surface reliefs along $X$ and $Y$ directions. Reproduced with permission. ${ }^{84}$ Copyright 2017, Elsevier. 
replica imprinted on a glass plate as the plasmon-enhanced near-field orbital AM light generator (the replica was also laminated with an azopolymer film); and the nanoscale mass transport of the azopolymer by the near-field orbital AM light. A right-handed circularly polarized 532-nm plane-wave light field was uniformly irradiated on this Au-coated replica covered with an azopolymer film from through the glass substrate with an optical power of $<5 \mathrm{~mW} / \mathrm{cm}^{2}$, which was significantly (ca. $10^{-5}$ times) less than the threshold power needed for the surface relief formation. In this regime, a right-handed chiral surface relief was formed in the azopolymer film with a
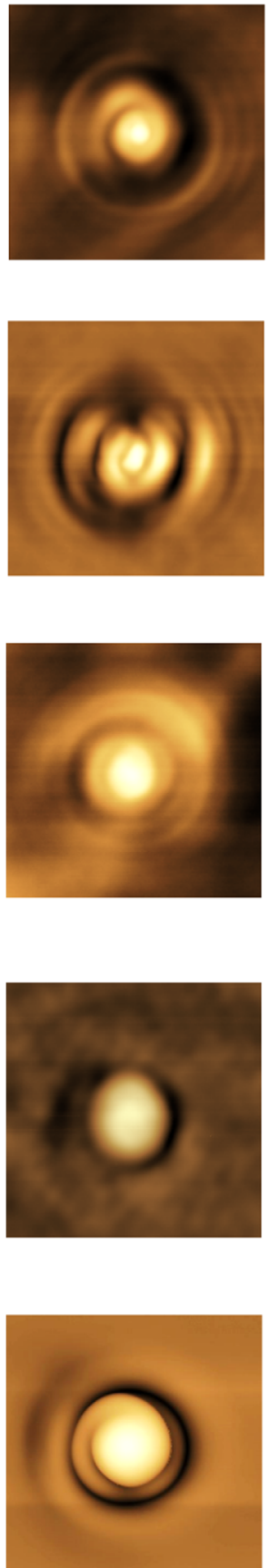

(a)

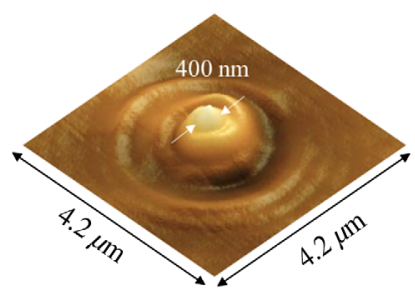

(b)

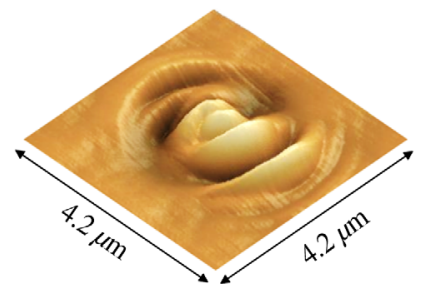

(c)

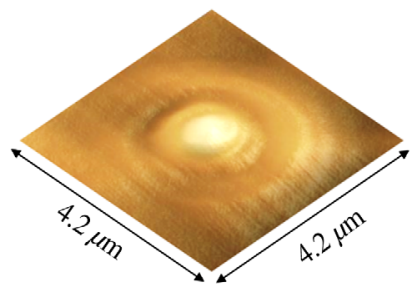

(d)
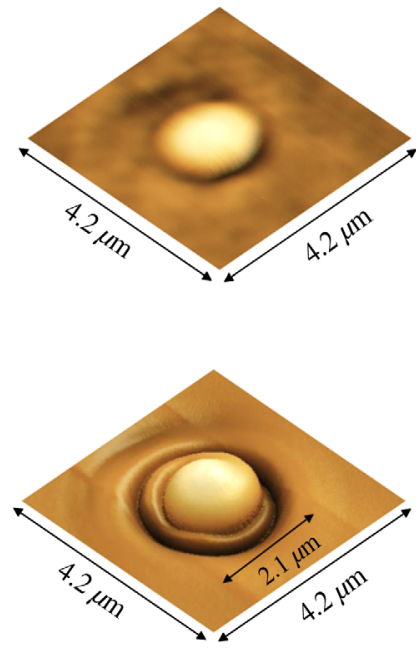

(e)

Fig. 16 Chiral surface relief formed on the azopolymer film. (a) Right-handed-, (b) linearly- and (c) left-handed polarized 532-nm light for an exposure time of $10 \mathrm{~min}$. (d) Right-handed circularly polarized light was irradiated on the device without Au thin film. (e) Original chiral surface relief in azopolymer film formed using a focused right-handed circularly polarized 532-nm optical vortex. Reproduced with permission. ${ }^{55}$ Copyright 2018, The Optical Society. 


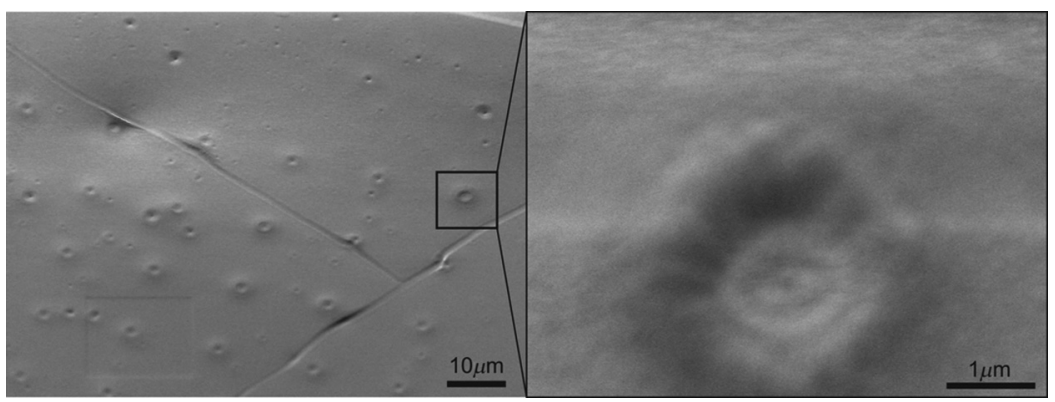

Fig. 17 SEM images of the surface relief profile on the azopolymer layer deposited on the inner surface of the hyperlens. Reproduced with permission. ${ }^{86}$ Copyright 2018, SPIE.

diameter of ca. $400 \mathrm{~nm}$, which is around $15 \%$ to $20 \%$ of the size of the original relief, as shown in Fig. 16(a). In contrast, the use of left-handed circularly polarized light or linearly polarized plane-wave light prevented such nanoscale chiral mass transport of the laminated azopolymer, as shown in Fig. 16(b). As a result, a shallow nonchiral surface relief was formed in the azopolymer film. It is also noteworthy that no chiral relief was formed without an Au film, even with right-handed circularly polarized light [Fig. 16(c)].

Thus, these phenomena are induced by the plasmon-enhanced near-field orbital AM light, which is caused by the spin-orbital AM coupling between a circularly polarized beam and an Au-coated replica of chiral surface relief.

A micrometer-scale vortex mode converter with a high demagnification rate hyperlens is capable of generating optical fields with orbital AM on the subwavelength scale below the diffraction limit. ${ }^{85,86}$ The hyperlens is a curved hyperbolic metamaterial consisting of a metal/ dielectric multilayered structure, enabling the conversion between the evanescent wave components propagating waves. ${ }^{47}$ A circularly polarized Gaussian light beam from a 405-nm laser source was converted into a radially polarized vortex beam using a vortex converter that consisted of concentric rings of $\mathrm{Cr}$ integrated on the outer surface of the hyperlens. The beam was then demagnified by a hyperlens formed of 26 alternating layers of $\mathrm{Ag}$ and $\mathrm{Ti}_{3} \mathrm{O}_{5}$ to be an annular spot with a diameter of $300 \mathrm{~nm}$. Surface relief formation in the azopolymer film was performed by the focused radially polarized vortex without the hyperlens using an objective lens (NA 0.3), which resulted in the formation of a nonchiral surface relief identical to that formed on the inner surface of the hyperlens. These results indicate that the hyperlens enabled demagnification of the radially polarized orbital AM beam down to nanoscale dimensions and the nanoscale mass transport of azopolymers ${ }^{86}$ (Fig. 17).

\section{Conclusion}

Optical light fields with orbital AM are associated with their helical wavefront and typically possess on-axial phase singularities. Their orbital AM enables materials to be twisted, so that a variety of chiral structures can be shaped at the nano-/microscale. In particular, azopolymers exhibit some of the most striking, diverse structures and phenomena in the formation of a surface relief, whose chirality can be selectively controlled by the handedness of the orbital AM light field, i.e., the sign of the topological charge of the field. When accompanied with spin AM associated with the rotating electric field of the circular polarized light, spin AM and orbital AM can be coupled, manifesting new fundamental physical consequences, such as the degeneracy breaking of the orbital AM fields, even with the same total AM in the surface relief formation.

Structured materials that are nonexistent in nature, such as a hyperlens and a chiral surface relief itself, should potentially enable the generation of nanoscale orbital AM fields, which can thus induce chiral mass transport of azopolymers and form further nanoscale chiral surface reliefs in azopolymers. These nanoscale chiral mass transport induced by illumination with a nanoscale optical field with orbital AM should also open the new route to the development of a broad range of advanced nanomaterials of current interest in photonics, e.g., metasurfaces for selective detection of chiral chemical composites and control of chiral chemical reactions. 


\section{Acknowledgments}

The authors acknowledge support in the form of KAKENHI Grants-in-Aid (Grant Nos. JP 16H06507, JP 17K19070, and JP 18H03884) from the Japan Society for the Promotion of Science (JSPS), Japan Science and Technology Agency (JST) CREST Grant No. (JPMJCR1903), and the U.S. National Science Foundation Award \#1809518. KD and YA thank the UK Engineering and Physical Sciences Research Council for funding (through Grant No. $\mathrm{EP} / \mathrm{P} 030017 / 1)$.

\section{References}

1. L. Allen et al., "Orbital angular momentum of light and the transformation of LaguerreGaussian laser modes," Phys. Rev. A 45(11), 8185-8189 (1992).

2. M. Padgett, J. Courtial, and L. Allen, "Light's orbital angular momentum," Phys. Today 57(5), 35-40 (2004).

3. M. J. Padgett, "Orbital angular momentum 25 years on [Invited]," Opt. Express 25(10), 11265-11274 (2017).

4. T. Kuga et al., "Novel optical trap of atoms with a doughnut beam," Phys. Rev. Lett. 78(25), 4713-4716 (1997).

5. M. Padgett and R. Bowman, "Tweezers with a twist," Nat. Photonics 5(6), 343-348 (2011).

6. D. G. Grier, “A revolution in optical manipulation," Nature 424(6950), 810-816 (2003).

7. K. Dholakia and T. Čižmár, "Shaping the future of manipulation," Nat. Photonics 5(6), 335-342 (2011).

8. I. Heller et al., "STED nanoscopy combined with optical tweezers reveals protein dynamics on densely covered DNA," Nat. Methods 10(9), 910-916 (2013).

9. M. Kamper et al., "Near-infrared STED nanoscopy with an engineered bacterial phytochrome," Nat. Commun. 9(1), 4762 (2018).

10. Y. Iketaki et al., "Investigation of the fluorescence depletion process in the condensed phase; application to a tryptophan aqueous solution," Chem. Phys. Lett. 372(5-6), 773-778 (2003).

11. G. Molina-Terriza, J. P. Torres, and L. Torner, "Twisted photons," Nat. Phys. 3(5), 305-310 (2007).

12. N. Bozinovic et al., "Multiplexing in fibers," Science 340(6140), 1545-1548 (2013).

13. J. Wang, "Advances in communications using optical vortices," Photonics Res. 4(5), B14 (2016)

14. Y. Li, L. Yu, and Y. Zhang, "Influence of anisotropic turbulence on the orbital angular momentum modes of Hermite-Gaussian vortex beam in the ocean," Opt. Express 25(11), 12203-12215 (2017).

15. Y. Huang et al., "Evolution behavior of Gaussian Schell-model vortex beams propagating through oceanic turbulence," Opt. Express 22(15), 17723-17734 (2014).

16. K. Toyoda et al., "Using optical vortex to control the chirality of twisted metal nanostructures," Nano Lett. 12(7), 3645-3649 (2012).

17. K. Toyoda et al., "Transfer of light helicity to nanostructures," Phys. Rev. Lett. 110(14), 143603 (2013).

18. T. Omatsu et al., "Metal microneedle fabrication using twisted light with spin," Opt. Express 18(17), 17967-17973 (2010).

19. S. Syubaev et al., "Zero-orbital-angular-momentum laser printing of chiral nanoneedles," Opt. Lett. 42(23), 5022-5025 (2017).

20. S. Syubaev et al., "Direct laser printing of chiral plasmonic nanojets by vortex beams," Opt. Express 25(9), 10214-10223 (2017).

21. F. Takahashi et al., "Optical vortex pulse illumination to create chiral monocrystalline silicon nanostructures," Phys. Status Solidi Appl. Mater. Sci. 213(4), 1063-1068 (2016).

22. F. Takahashi et al., "Picosecond optical vortex pulse illumination forms a monocrystalline silicon needle," Sci. Rep. 6, 21738 (2016).

23. S. M. Barnett, "Optical angular-momentum flux," J. Opt. B Quantum Semiclassical Opt. 4, S7-S16 (2002). 
24. A. T. O'Neil et al., "Intrinsic and extrinsic nature of the orbital angular momentum of a light beam," Phys. Rev. Lett. 88(5), 053601 (2002).

25. J. Leach et al., "Interferometric methods to measure orbital and spin, or the total angular momentum of a single photon," Phys. Rev. Lett. 92(1), 013601 (2004).

26. V. Garcés-Chávez et al., "Observation of the transfer of the local angular momentum density of a multiringed light beam to an optically trapped particle," Phys. Rev. Lett. 91(9), 093602 (2003).

27. H. He et al., "Direct observation of transfer of angular momentum to absorptive particles from a laser beam with a phase singularity," Phys. Rev. Lett. 75(5), 826-829 (1995).

28. S. M. Barnett et al., "On the natures of the spin and orbital parts of optical angular momentum," J. Opt. 18(6), 064004 (2016).

29. T. Omatsu, K. Miyamoto, and R. Morita, "Optical vortices illumination enables the creation of chiral nanostructures," in Vortex Dynamics and Optical Vortices, Intech open 4, H. P. De Tejada, Ed., p. 119, InTech, London, United Kingdom (2017).

30. S. Kawagoe et al., "Microfabrication of Au film using optical vortex beam," J. Laser Micro/ Nanoeng. 14(1), 31-34 (2019).

31. A. S. Kewitsch and A. Yariv, "Self-focusing and self-trapping of optical beams upon photopolymerization," Opt. Lett. 21(1), 24-26 (1996).

32. J. Ježek et al., "Formation of long and thin polymer fiber using nondiffracting beam," Opt. Express 14(19), 8506-8515 (2006).

33. J. Lee et al., "Photopolymerization with light fields possessing orbital angular momentum: generation of helical micro fibers," ACS Photonics 5, 4156-4163 (2018).

34. W. Man et al., "Optical nonlinearities and enhanced light transmission in soft-matter systems with tunable polarizabilities," Phys. Rev. Lett. 111(21), 218302 (2013).

35. J. Sun et al., "Nanoscale orbital angular momentum beam instabilities in engineered nonlinear colloidal media," Opt. Express 26(5), 5118-5125 (2018).

36. K. Y. Bliokh et al., "Spin-orbit interactions of light," Nat. Photonics 9(12), 796-808 (2015).

37. W. Tsai, J. Huang, and C. Huang, "Selective trapping or rotation of isotropic dielectric microparticles by optical near field in a plasmonic archimedes spiral," Nano Lett. 14(2), 547-552 (2014).

38. G. Spektor et al., "Revealing the subfemtosecond dynamics of orbital angular momentum in nanoplasmonic vortices," Science 355(6330), 1187-1191 (2017).

39. S. L. Cheng, C. Y. Chen, and S. W. Lee, "Kinetic investigation of the electrochemical synthesis of vertically-aligned periodic arrays of silicon nanorods on (001)Si substrate," Thin Solid Films 518(6 Suppl. 1), S190-S195 (2010).

40. L. Torsi et al., "A sensitivity-enhanced field-effect chiral sensor," Nat. Mater. 7(5), 412-417 (2008).

41. A. E. Willner et al., "Optical communications using orbital angular momentum beams," Adv. Opt. Photonics 7(1), 66-98 (2015).

42. K. E. Chong et al., "Polarization-independent silicon metadevices for efficient optical wavefront control," Nano Lett. 15(8), 5369-5374 (2015).

43. J. Sun et al., "Spinning light on the nanoscale," Nano Lett. 14(5), 2726-2729 (2014).

44. Y. Wang et al., "Dynamically sculpturing plasmonic vortices: from integer to fractional orbital angular momentum," Sci. Rep. 6, 36269 (2016).

45. A. D. Mayevsky et al., "Mesoscale surface plasmons: modelling and imaging using nearfield scanning optical microscopy," Opt. Express 26(18), 23426-23435 (2018).

46. D. Stellinga et al., "An organic vortex laser," ACS Nano 12(3), 2389-2394 (2018).

47. J. Sun and N. M. Litchinitser, "Toward practical, subwavelength, visible-light photolithography with hyperlens," ACS Nano 12(1), 542-548 (2018).

48. Z. Sekkat, "Optical tweezing by photomigration," Appl. Opt. 55(2), 259-268 (2016).

49. J. Kumar et al., "Gradient force: the mechanism for surface relief grating formation in azobenzene functionalized polymers," Appl. Phys. Lett. 72(17), 2096-2098 (1998).

50. M. Warner and L. Mahadevan, "Photoinduced deformations of beams, plates, and films," Phys. Rev. Lett. 92(13), 134302 (2004).

51. N. K. Viswanathan et al., "Surface relief structures on azo polymer films," J. Mater. Chem. 9(9), 1941-1955 (1999). 
52. M. Watabe et al., "Light induced conch-shaped relief in an azo-polymer film," Sci. Rep. 4, 4281 (2015).

53. D. Barada et al., "Constructive spin-orbital angular momentum coupling can twist materials to create spiral structures in optical vortex illumination," Appl. Phys. Lett. 108(5), 051108 (2016).

54. K. Masuda et al., "Azo-polymer film twisted to form a helical surface relief by illumination with a circularly polarized Gaussian beam," Opt. Express 25(11), 12499-12507 (2017).

55. K. Masuda et al., "Nanoscale chiral surface relief of azo-polymers with nearfield OAM light," Opt. Express 26(17), 22197-22207 (2018).

56. A. Ambrosio et al., "Light-induced spiral mass transport in azo-polymer films under vortexbeam illumination," Nat. Commun. 3, 989 (2012).

57. A. Ambrosio, P. Maddalena, and L. Marrucci, "Molecular model for light-driven spiral mass transport in azopolymer films," Phys. Rev. Lett. 110(14), 1-5 (2013).

58. H. Ishitobi et al., "The anisotropic nanomovement of azo-polymers," Opt. Express 15(2), 652-659 (2007).

59. C. J. Barrett, A. L. Natansohn, and P. L. Rochon, "Mechanism of optically inscribed highefficiency diffraction gratings in azo polymer films," J. Phys. Chem. 100(21), 8836-8842 (1996).

60. P. Rochon, E. Batalla, and A. Natansohn, "Optically induced surface gratings on azoaromatic polymer films," Appl. Phys. Lett. 66(2), 136-138 (1995).

61. A. Kravchenko et al., "Optical interference lithography using azobenzene-functionalized polymers for micro-and nanopatterning of silicon," Adv. Mater. 23(36), 4174-4177 (2011).

62. H. Rau, "Photoisomerization of benzenes," in Photoreactive Organic Thin Films, Z. Sekkat and W. Knoll, Eds., pp. 3-28, Academic Press, Cambridge, Massachusetts (2002).

63. Z. Sekkat, "Photo-orientation by photoisomerization," in Photoreactive Organic Thin Films, Z. Sekkat and W. Knoll, Eds., pp. 64-102, Academic Press, Cambridge, Massachusetts (2002).

64. X. Wang, "Trans-cis isomerization," in Azo Polymers, First, X. Wang, Ed., pp. 19-56, Springer, Berlin, Heidelberg, Germany (2016).

65. T. Fujino, S. Y. Arzhantsev, and T. Tahara, "Femtosecond time-resolved fluorescence study of photoisomerization of trans-azobenzene," J. Phys. Chem. A 105(35), 8123-8129 (2001).

66. Z. Sekkat, J. Wood, and W. Knoll, "Reorientation mechanism of azobenzenes within the trans $\Rightarrow$ cis photoisomerization," J. Phys. Chem. 99(47), 17226-17234 (1995).

67. K. Yang, S. Yang, and J. Kumar, "Formation mechanism of surface relief structures on amorphous azopolymer films," Phys. Rev. B 73, 165204 (2006).

68. C. J. Barrett, P. L. Rochon, and A. L. Natansohn, "Model of laser-driven mass transport in thin films of dye-functionalized polymers," J. Chem. Phys. 109(4), 1505-1516 (1998).

69. P. Lefin, C. Fiorini, and J. M. Nunzi, "Anisotropy of the photoinduced translation diffusion of azo-dyes," Opt. Mater. 9(1-4), 323-328 (1998).

70. B. Bellini et al., "Light-induced molecular motion of azobenzene-containing molecules: a random-walk model," J. Phys. Condens. Matter 18(33), S1817-S1835 (2006).

71. T. G. Pedersen et al., "Mean-field theory of photoinduced formation of surface reliefs in side-chain azobenzene polymers," Phys. Rev. Lett. 80(1), 89-92 (1998).

72. K. Sumaru et al., "Photoinduced surface relief gratings on azopolymer films: analysis by a fluid mechanics model," Appl. Phys. Lett. 75(13), 1878-1880 (1999).

73. K. Sumaru et al., "Photoinduced surface relief formation on azopolymer films: a driving force and formed relief profile," J. Appl. Phys. 91(5), 3421-3430 (2002).

74. S. Bian et al., "Single laser beam-induced surface deformation on azobenzene polymer films," Appl. Phys. Lett. 73(13), 1817-1819 (1998).

75. S. Bian et al., "Photoinduced surface deformations on azobenzene polymer films," J. Appl. Phys. 86(8), 4498-4508 (1999).

76. Y. Zhao et al., "Spin-to-orbital angular momentum conversion in a strongly focused optical beam," Phys. Rev. Lett. 99(7), 15-18 (2007).

77. T. A. Nieminen et al., "Angular momentum of a strongly focused Gaussian beam," J. Opt. A 10(11), 115005 (2008). 
78. Y. Nakata, M. Yoshida, and N. Miyanaga, "Parallel fabrication of spiral surface structures by interference pattern of circularly polarized beams," Sci. Rep. 8(1), 13448 (2018).

79. S. W. Magennis et al., "Two-photon-induced photoisomerization of an azo dye," Chem. Mater. 17(8), 2059-2062 (2005).

80. R. Hagen and T. Bieringer, "Photoaddressable polymers for optical data storage," $A d v$. Mater. 13(23), 1805-1810 (2001).

81. H. Ishitobi et al., "Two-photon induced polymer nanomovement," Opt. Express 16(18), 14106-14114 (2008).

82. A. Ambrosio et al., "Real-time monitoring of the surface relief formation on azo-polymer films upon near-field excitation," J. Microsc. 229(2), 307-312 (2008).

83. P. Karageorgiev et al., "From anisotropic photo-fluidity towards nanomanipulation in the optical near-field," Nat. Mater. 4(9), 699-703 (2005).

84. H. Ishitobi et al., "Near-field optical mapping of single gold nano particles using photoinduced polymer movement of azo-polymers," Opt. Сommun. 387, 24-29 (2017).

85. X. Ma et al., "A planar chiral meta-surface for optical vortex generation and focusing," Sci. Rep. 5, 10365 (2015).

86. J. Sun et al., "Shrinking optical vortex to the nanoscale," Proc. SPIE 10712, 107120T (2018).

Takashige Omatsu has been developing optical vortex sources and pioneering nano/micro structures fabrication based on optical vortex illumination, in Chiba University. He is currently serving as the founding editor-in-chief, OSA Continuum, OSA Publishing. He was awarded a prize for Science and Technology, MEXT, Japan. He was also elected as OSA Fellow, SPIE Fellow, and JSAP Fellow.

Kishan Dholakia is a professor of physics at the University of St. Andrews, Scotland. He works on advanced optical beam shaping for biophotonics, precision measurement, and optical manipulation. He has over 300 refereed journal papers and in excess of 29,000 citations (Google Scholar). He is recipient of the R.W. Wood Prize of the Optical Society (2016), the IOP Thomas Young Medal and Prize (2017) and the SPIE Dennis Gabor Award (2018).

Biographies of the other authors are not available. 